\title{
BARTONELLA ENDOCARDITIS LEADING TO ACUTE DIFFUSE GLOMERULONEPHRITIS AND HIGH LEVELS OF PR3-ANCA POSITIVITE
}

Jean Michell Correia Monteiro ${ }^{1, *}$, Nestor José de Souza Barreto Neto ${ }^{1}$, Luma Mendes Brito ${ }^{1}$, Henrique Ayres Mayrink Giardini ${ }^{1}$, Lissiane Karine Noronha Guedes ${ }^{1}$, Rosa Maria Rodrigues Pereira ${ }^{1}$

1. Universidade de São Paulo, São Paulo (SP), Brazil.

*Corresponding author:jk_michell@hotmail.com

\section{BACKGROUND}

Bartonella infection is a recognized cause of culture-negative endocarditis. As other causes of endocarditis, this infection may also trigger immunologic responses leading to systemic inflammation, mimicking ANCA-associated vasculitides.

\section{CASE REPORT}

A 45-year-old man with a two-year history of bariatric surgery was admitted to our service for investigation of ANCA-associated vasculitis with endocarditis. One month before admission, he started to present symmetrical edema of the lower limbs and purpuric lesions, as well as dyspnea and fatigue, having been submitted to echocardiography which demonstrated valve vegetation. He thus received the diagnosis of endocarditis, though blood cultures were persistently negative. The patient also had dysmorphic hematuria and subnephrotic proteinuria and was submitted to renal biopsy, which was compatible with post-infectious acute diffuse glomerulonephritis. He received antibiotic therapy and was discharged with prednisone $1 \mathrm{mg} / \mathrm{kg} / \mathrm{day}$. He was also referred for rheumatology evaluation due to the presence of positive c-ANCA. Despite treatment, the patient maintained weight loss and overall clinical worsening, and was admitted in the rheumatology service for etiological investigation. He maintained active urinary sediment and repeated negative blood cultures. Complement C3 and C4 were slightly consumed. Tests for HIV, hepatitis B and C were negative, but he had a positive treponemal test and VDRL of 1:80. Specific autoantibodies were negative. The ANCA indirect immunofluorescence was negative, but anti-PR3 was positive in high levels (147-reference range $<20$ ). Antimyeloperoxidase (MPO) was negative (4 - reference range $<20$ ). A new echocardiogram confirmed mitral and aortic valve endocarditis, with severe aortic valve insufficiency. Initial evaluation for occult neoplasia was negative. Serology for Bartonella spp. was positive. Treatment with ceftriaxone, doxycycline and gentamicin was initiated, but the patient developed digestive hemorrhage requiring hospitalization in the intensive care unit, with worsening renal function and need for hemodialysis. After clinical compensation, he underwent aortic valve replacement and mitral valve repair. He was eventually discharged after 140 days of hospitalization for motor rehabilitation and completion of targeted antibiotic treatment with recovery of renal function.

\section{CONCLUSION}

We interpret the immunological responses seen in this patient as a result of Bartonella infection, rather than primary ANCAvasculitis, as this bacterium is typically associated with ANCA positivity and the pattern of glomerulonephritis observed resembles a postinfectious condition. Therefore, blood cultures, echocardiogram, and other means of investigation for endocarditis must be considered in patients with systemic inflammation and positive ANCA tests. 